\title{
Mitochondrial Mechanisms and Neurological Disorders
}

\author{
Andrea L. Gropman
}

Published online: 27 February 2013

(C) The American Society for Experimental NeuroTherapeutics, Inc. 2013

It is increasingly recognized that altered mitochondrial function can contribute to the pathobiology of many neurologic diseases. This special issue of Neurotherapeutics provides a critical review of recent developments in this area.

Mitochondria play an important role in oxidative phosphorylation and energy production. Myriad disorders result from impaired energy production in both children and adults. Mitochondrial disorders are often very difficult to recognize, diagnose, and treat. In part, this relates to the marked clinical variability of such disorders, which involve multiple organ systems.

Over the past 20 years there have been marked advances in the understanding of mitochondrial genetics, as well as mitochondrial changes that contribute to neurologic disease states. In addition, the variability of clinical phenotype has been underscored, along with the recognition that mitochondrial disorders may present differently in children and adults.

This issue includes 11 critical reviews written by leading authorities in mitochondrial disease. The first section provides descriptive reviews of mitochondrial disorders and their clinical characteristics. Saneto et al. (see pages
$\mathrm{XXX}$ ), describe mtDNA-encoded disorders in children, Goldstein et al. (see pages XXX) discuss nuclear encoded disorders, and El-Hattab and Scaglia (pages XXX) address mitochondrial depletion disorders. Adult-onset mitochondrial disorders are reviewed by Cohen (pages XXX), and counseling-related issues by Vento and Pappa (pages XXX).

The second section reviews imaging biomarkers by Gropman (pages XXX) and diagnostic methods by Falk and Wong (pages XXX), including next generation sequencing methods. The final section addresses potential therapeutics, with articles by Wolfe (pages XXX), Kerr (pages XXX), and Hurko (pages XXX). The latter includes an overview of clinical trials and potential biological therapies.

Space limitations did not permit a truly comprehensive review of this rapidly expanding field. Nonetheless, it is hoped that this publication may serve as an important reference issue that stimulates further research in this important area

Required Author Forms Disclosure forms provided by the authors are available with the online version of this article.

A. L. Gropman

Neurodevelopmental Pediatrics and Neurogenetics, Children's

National Medical Center, 111 Michigan Avenue, N.W.,

Washington, DC 20010, USA

\author{
A. L. Gropman ( $ه)$ \\ The George Washington University of the Health Sciences, \\ Washington, DC, USA \\ e-mail: agropman@childrensnational.org
}

